\title{
Inner Regularization of Log-Concave Measures and Small-Ball Estimates
}

\author{
Bo'az Klartag ${ }^{1}$ and Emanuel Milman ${ }^{2}$
}

\begin{abstract}
In the study of concentration properties of isotropic log-concave measures, it is often useful to first ensure that the measure has super-Gaussian marginals. To this end, a standard preprocessing step is to convolve with a Gaussian measure, but this has the disadvantage of destroying small-ball information. We propose an alternative preprocessing step for making the measure seem super-Gaussian, at least up to reasonably high moments, which does not suffer from this caveat: namely, convolving the measure with a random orthogonal image of itself. As an application of this "inner-thickening", we recover Paouris' small-ball estimates.
\end{abstract}

\section{Introduction}

Fix a Euclidean norm $|\cdot|$ on $\mathbb{R}^{n}$, and let $X$ denote an isotropic random vector in $\mathbb{R}^{n}$ with log-concave density $g$. Recall that a random vector $X$ in $\mathbb{R}^{n}$ (and its density) is called isotropic if $\mathbb{E} X=0$ and $\mathbb{E} X \otimes X=I d$, i.e. its barycenter is at the origin and its covariance matrix is equal to the identity one. Taking traces, we observe that $\mathbb{E}|X|^{2}=n$. Here and throughout we use $\mathbb{E}$ to denote expectation and $\mathbb{P}$ to denote probability. A function $g: \mathbb{R}^{n} \rightarrow \mathbb{R}_{+}$is called log-concave if $-\log g: \mathbb{R}^{n} \rightarrow \mathbb{R} \cup\{+\infty\}$ is convex. Throughout this work, $C, c, c_{2}, C^{\prime}$, etc. denote universal positive numeric constants, independent of any other parameter and in particular the dimension $n$, whose value may change from one occurrence to the next.

Any high-dimensional probability distribution which is absolutely continuous has at least one super-Gaussian marginal (e.g. [13]). Still, in the study of concentration properties of $X$ as above, it is many times advantageous to know that all of the onedimensional marginals of $X$ are super-Gaussian, at least up to some level (see e.g. [24, 9, 14]). By this we mean that for some $p_{0} \geq 2$ :

$$
\forall 2 \leq p \leq p_{0} \quad \forall \theta \in S^{n-1} \quad\left(E|\langle X, \theta\rangle|^{p}\right)^{\frac{1}{p}} \geq c\left(E\left|G_{1}\right|^{p}\right)^{\frac{1}{p}},
$$

\footnotetext{
${ }^{1}$ School of Mathematical Sciences, Tel-Aviv University, Tel Aviv 69978, Israel. Supported in part by the Israel Science Foundation and by a Marie Curie Reintegration Grant from the Commission of the European Communities. Email: klartagb@tau.ac.il

${ }^{2}$ Department of Mathematics, Technion - Israel Institute of Technology, Haifa 32000, Israel. Supported by ISF, GIF, the Taub Foundation (Landau Fellow) and the E. and J. Bishop Research Fund. Email: emilman@tx.technion.ac.il.
} 
where $G_{1}$ denotes a one-dimensional standard Gaussian random variable and $S^{n-1}$ is the Euclidean unit sphere in $\mathbb{R}^{n}$. It is convenient to reformulate this using the language of $L_{p}$-centroid bodies, which were introduced by E. Lutwak and G. Zhang in [16] (under a different normalization). Given a random vector $X$ with density $g$ on $\mathbb{R}^{n}$ and $p \geq 1$, the $L_{p}$-centroid body $Z_{p}(X)=Z_{p}(g) \subset \mathbb{R}^{n}$ is the convex set defined via its support functional $h_{Z_{p}(X)}$ by:

$$
h_{Z_{p}(X)}(y)=\left(\int_{\mathbb{R}^{n}}|\langle x, y\rangle|^{p} g(x) d x\right)^{1 / p}, y \in \mathbb{R}^{n} .
$$

More generally, the one-sided $L_{p}$-centroid body, denoted $Z_{p}^{+}(X)$, was defined in [9] (cf. [10]) by:

$$
h_{Z_{p}^{+}(X)}(y)=\left(2 \int_{\mathbb{R}^{n}}\langle x, y\rangle_{+}^{p} g(x) d x\right)^{1 / p}, y \in \mathbb{R}^{n},
$$

where as usual $a_{+}:=\max (a, 0)$. Note that when $g$ is even then both definitions above coincide, and that when the barycenter of $X$ is at the origin, $Z_{2}(X)$ is the Euclidean ball $B_{2}^{n}$ if and only $X$ is isotropic. Observing that the right-hand side of (1.1) is of the order of $\sqrt{p}$, we would like to have:

$$
\forall 2 \leq p \leq p_{0} \quad Z_{p}^{+}(X) \supset c \sqrt{p} B_{2}^{n},
$$

where $B_{2}^{n}=\left\{x \in \mathbb{R}^{n} ;|x| \leq 1\right\}$ is the unit Euclidean ball.

Unfortunately, we cannot in general expect to satisfy (1.2) for $p_{0}$ which grows with the dimension $n$. This is witnessed by $X$ which is uniformly distributed on the $n$-dimensional cube $[-\sqrt{3}, \sqrt{3}]^{n}$ (the normalization ensures that $X$ is isotropic), whose marginals in the directions of the axes are uniform on a constant-sized interval. Consequently, some preprocessing on $X$ is required, which on one hand transforms it into another random variable $Y$ whose density $g$ satisfies (1.2), and on the other enables deducing back the desired concentration properties of $X$ from those of $Y$.

A very common such construction is to convolve with a Gaussian, i.e. define $Y:=$ $\left(X+G_{n}\right) / \sqrt{2}$, where $G_{n}$ denotes an independent standard Gaussian random vector in $\mathbb{R}^{n}$. In [11] (and in subsequent works like [12, 5]), the Gaussian played more of a regularizing role, but in [9], its purpose was to "thicken from inside" the distribution of $X$, ensuring that (1.2) is satisfied for all $p \geq 2$ (see [9, Lemma 2.3]). Regarding the transference of concentration properties, it follows from the argument in the proof of [11, Proposition 4.1] that:

$$
\mathbb{P}(|X| \geq(1+t) \sqrt{n}) \leq C \mathbb{P}\left(|Y| \geq \sqrt{\frac{(1+t)^{2}+1}{2}} \sqrt{n}\right) \quad \forall t \geq 0,
$$

and:

$$
\mathbb{P}(|X| \leq(1-t) \sqrt{n}) \leq C \mathbb{P}\left(|Y| \leq \sqrt{\frac{(1-t)^{2}+1}{2}} \sqrt{n}\right) \quad \forall t \in[0,1]
$$


for some universal constant $C>1$. The estimate (1.3) is perfectly satisfactory for transferring (after an adjustment of constants) deviation estimates above the expectation from $|Y|$ to $|X|$. However, note that the right-hand side of (1.4) is bounded below by $P(|Y| \leq \sqrt{n / 2}$ ) (and in particular does not decay to 0 when $t \rightarrow 1$ ), and so (1.4) is meaningless for transferring small-ball estimates from $|Y|$ to $|X|$. Consequently, the strategies employed in [11, 12, 5, 9] did not and could not deduce the concentration properties of $|X|$ in the small-ball regime. This seems an inherent problem of adding an independent Gaussian: small-ball information is lost due to the "Gaussian-thickening".

The purpose of this note is to introduce a different inner-thickening step, which does not have the above mentioned drawback. Before formulating it, recall that $X$ (or its density) is said to be " $\psi_{\alpha}$ with constant $D>0$ " if:

$$
Z_{p}(X) \subset D p^{1 / \alpha} Z_{2}(X) \quad \forall p \geq 2
$$

We will simply say that " $X$ is $\psi_{\alpha}$ ", if it is $\psi_{\alpha}$ with constant $D \leq C$, and not specify explicitly the dependence of the estimates on the parameter $D$. By a result of Berwald [1] (or applying Borell's Lemma [3] as in [21, Appendix III]), it is well known that any $X$ with $\log$-concave density satisfies:

$$
1 \leq p \leq q \quad \Rightarrow \quad Z_{p}(X) \subset Z_{q}(X) \subset C \frac{q}{p} Z_{p}(X)
$$

In particular, such an $X$ is always $\psi_{1}$ with some universal constant, and so we only gain additional information when $\alpha>1$.

Theorem 1.1. Let $X$ denote an isotropic random vector in $\mathbb{R}^{n}$ with a log-concave density, which is in addition $\psi_{\alpha}(\alpha \in[1,2])$, and let $X^{\prime}$ denote an independent copy of $X$. Given $U \in O(n)$, the group of orthogonal linear maps in $\mathbb{R}^{n}$, denote:

$$
Y_{ \pm}^{U}:=\frac{X \pm U\left(X^{\prime}\right)}{\sqrt{2}}
$$

Then:

1. For any $U \in O(n)$, the concentration properties of $\left|Y_{ \pm}^{U}\right|$ are transferred to $|X|$ as follows:

$P(|X| \geq(1+t) \sqrt{n}) \leq\left(2 \max \left(P\left(\left|Y_{+}^{U}\right| \geq(1+t) \sqrt{n}\right), P\left(\left|Y_{-}^{U}\right| \geq(1+t) \sqrt{n}\right)\right)\right)^{1 / 2} \quad \forall t \geq 0$,

and:

$P(|X| \leq(1-t) \sqrt{n}) \leq\left(2 \max \left(P\left(\left|Y_{+}^{U}\right| \leq(1-t) \sqrt{n}\right), P\left(\left|Y_{-}^{U}\right| \leq(1-t) \sqrt{n}\right)\right)\right)^{1 / 2} \quad \forall t \in[0,1]$.

2. For any $U \in O(n)$ :

$$
Z_{p}^{+}\left(Y_{ \pm}^{U}\right) \subset C p^{1 / \alpha} B_{2}^{n} \quad \forall p \geq 2
$$


3. There exists a subset $A \subset O(n)$ with:

$$
\mu_{O(n)}(A) \geq 1-\exp (-c n)
$$

where $\mu_{O(n)}$ denotes the Haar measure on $O(n)$ normalized to have total mass 1 , so that if $U \in A$ then:

$$
Z_{p}^{+}\left(Y_{ \pm}^{U}\right) \supset c_{1} \sqrt{p} B_{2}^{n} \quad \forall p \in\left[2, c_{2} n^{\frac{\alpha}{2}}\right]
$$

Remark 1.2. Note that when the density of $X$ is even, then $Y_{+}^{U}$ and $Y_{-}^{U}$ in Theorem 1.1 are identically distributed, which renders the formulation of the conclusion more natural. However, we do not know how to make the formulation simpler in the non-even case.

Remark 1.3. Also note that $Y_{ \pm}^{U}$ are isotropic random vectors, and that by the PrékopaLeindler Theorem (e.g. [7]), they have log-concave densities.

As our main application, we manage to extend the strategy in the second named author's previous work with O. Guédon [9] to the small-ball regime, and obtain:

Corollary 1.4. Let $X$ denote an isotropic random vector in $\mathbb{R}^{n}$ with log-concave density, which is in addition $\psi_{\alpha}(\alpha \in[1,2])$. Then:

$$
\mathbb{P}(|| X|-\sqrt{n}| \geq t \sqrt{n}) \leq C \exp \left(-c n^{\frac{\alpha}{2}} \min \left(t^{2+\alpha}, t\right)\right) \quad \forall t \geq 0,
$$

and:

$$
\mathbb{P}(|X| \leq \varepsilon \sqrt{n}) \leq(C \varepsilon)^{c n^{\frac{\alpha}{2}}} \forall \varepsilon \in[0,1 / C]
$$

Corollary 1.4 is an immediate consequence of Theorem 1.1 and the following result, which is the content of [9, Theorem 4.1] (our formulation below is slightly more general, but this is what the proof gives):

Theorem (Guédon-Milman). Let $Y$ denote an isotropic random vector in $\mathbb{R}^{n}$ with a log-concave density, so that in addition:

$$
c_{1} \sqrt{p} B_{2}^{n} \subset Z_{p}^{+}(Y) \subset c_{2} p^{1 / \alpha} B_{2}^{n} \quad \forall p \in\left[2, c_{3} n^{\frac{\alpha}{2}}\right],
$$

for some $\alpha \in[1,2]$. Then (1.9) and (1.10) hold with $X=Y$ (and perhaps different constants $C, c>0$ ).

We thus obtain a preprocessing step which fuses perfectly with the approach in [9], allowing us to treat all deviation regimes simultaneously in a single unified framework. We point out that Corollary 1.4 by itself is not new. The large positive-deviation estimate:

$$
P(|X| \geq(1+t) \sqrt{n}) \leq \exp \left(-c n^{\frac{\alpha}{2}} t\right) \quad \forall t \geq C,
$$

was first obtained by G. Paouris in [22]; it is known to be sharp, up to the value of the constants. The more general deviation estimate (1.9) was obtained in [9], improving 
when $t \in[0, C]$ all previously known results due to the first named author and to Fleury [11, 12, 5] (we refer to [9] for a more detailed account of these previous estimates). In that work, the convolution with Gaussian preprocessing was used, and so it was not possible to independently deduce the small-ball estimate (1.10). The latter estimate was first obtained by Paouris in [23], using the reverse Blaschke-Santaló inequality of J. Bourgain and V. Milman [4]. In comparison, our main tool in the proof of Theorem 1.1 is a covering argument in the spirit of V. Milman's M-position [17, 19, 18] (see also [25]), together with a recent lower-bound on the volume of $Z_{p}$ bodies obtained in our previous joint work [14].

Acknowledgement. We thank Vitali Milman and Olivier Guédon for discussions.

\section{Key Proposition}

In this section, we prove the following key proposition:

Proposition 2.1. Let $X, X^{\prime}$ be as in Theorem 1.1, let $U$ be uniformly distributed on $O(n)$, and set:

$$
Y:=\frac{X+U\left(X^{\prime}\right)}{\sqrt{2}}
$$

Then there exists a $c>0$, so that:

$$
\forall C_{1}>0 \exists c_{1}>0 \quad \forall p \in\left[2, c n^{\alpha / 2}\right] \quad \mathbb{P}\left(Z_{p}^{+}(Y) \supset c_{1} \sqrt{p} B_{2}^{n}\right) \geq 1-\exp \left(-C_{1} n\right) .
$$

Here, as elsewhere, "uniformly distributed on $O(n)$ " is with respect to the probability measure $\mu_{O(n)}$.

We begin with the following estimate due to Grünbaum [8] (see also [6, Formula (10)] or [2, Lemma 3.3] for simplified proofs):

Lemma 2.2 (Grünbaum). Let $X_{1}$ denote a random variable on $\mathbb{R}$ with log-concave density and barycenter at the origin. Then $\frac{1}{e} \leq \mathbb{P}\left(X_{1} \geq 0\right) \leq 1-\frac{1}{e}$.

Recall that the Minkowski sum $K+L$ of two compact sets $K, L \subset \mathbb{R}^{n}$ is defined as the compact set given by $\{x+y ; x \in K, y \in L\}$. When $K, L$ are convex, the support functional satisfies $h_{K+L}=h_{K}+h_{L}$.

Lemma 2.3. With the same notations as in Proposition 2.1:

$$
Z_{p}^{+}(Y) \supset \frac{1}{2 \sqrt{2} e^{1 / p}}\left(Z_{p}^{+}(X)+U\left(Z_{p}^{+}(X)\right)\right) .
$$

Proof. Given $\theta \in S^{n-1}$, denote $Y_{1}=\langle Y, \theta\rangle, X_{1}=\langle X, \theta\rangle$ and $X_{1}^{\prime}=\left\langle U\left(X^{\prime}\right), \theta\right\rangle$. By the Prékopa-Leindler theorem (e.g. [7]), all these one-dimensional random variables have log-concave densities, and since their barycenter is at the origin, we obtain by Lemma 2.2 ,

$$
h_{Z_{p}^{+}(Y)}^{p}(\theta)=2 \mathbb{E}\left(Y_{1}\right)_{+}^{p}=\frac{2}{2^{p / 2}} \mathbb{E}\left(X_{1}+X_{1}^{\prime}\right)_{+}^{p} \geq \frac{2}{2^{p / 2}} \mathbb{E}\left(X_{1}\right)_{+}^{p} \mathbb{P}\left(X_{1}^{\prime} \geq 0\right) \geq \frac{2}{e 2^{p / 2}} \mathbb{E}\left(X_{1}\right)_{+}^{p} .
$$


Exchanging the roles of $X_{1}$ and $X_{1}^{\prime}$ above, we obtain:

$$
h_{Z_{p}^{+}(Y)}^{p}(\theta) \geq \frac{1}{e 2^{p / 2}} \max \left(h_{Z_{p}^{+}(X)}^{p}(\theta), h_{Z_{p}^{+}\left(U\left(X^{\prime}\right)\right)}^{p}(\theta)\right) .
$$

Consequently:

$$
h_{Z_{p}^{+}(Y)}(\theta) \geq \frac{1}{\sqrt{2} e^{1 / p}} \frac{h_{Z_{p}^{+}(X)}(\theta)+h_{Z_{p}^{+}\left(U\left(X^{\prime}\right)\right)}(\theta)}{2},
$$

and since $Z_{p}^{+}\left(U\left(X^{\prime}\right)\right)=U\left(Z_{p}^{+}\left(X^{\prime}\right)\right)=U\left(Z_{p}^{+}(X)\right)$, the assertion follows.

Next, recall that given two compact subsets $K, L \subset \mathbb{R}^{n}$, the covering number $N(K, L)$ is defined as the minimum number of translates of $L$ required to cover $K$. The volumeradius of a compact set $K \subset \mathbb{R}^{n}$ is defined as:

$$
\operatorname{V.Rad} .(K)=\left(\frac{\operatorname{Vol}(K)}{\operatorname{Vol}\left(B_{2}^{n}\right)}\right)^{\frac{1}{n}},
$$

measuring the radius of the Euclidean ball whose volume equals the volume of $K$. A convex compact set with non-empty interior is called a convex body, and given a convex body $K$ with the origin in its interior, its polar $K^{\circ}$ is the convex body given by:

$$
K^{\circ}:=\left\{y \in \mathbb{R}^{n} ;\langle x, y\rangle \leq 1 \quad \forall x \in K\right\} .
$$

Finally, the mean-width of a convex body $K$, denoted $W(K)$, is defined as $W(K)=$ $2 \int_{S^{n-1}} h_{K}(\theta) d \mu_{S^{n-1}}(\theta)$, where $\mu_{S^{n-1}}$ denotes the Haar probability measure on $S^{n-1}$. The following two lemmas are certainly well-known; we provide a proof for completeness.

Lemma 2.4. Let $K \subset \mathbb{R}^{n}$ be a convex body with barycenter at the origin, so that:

$$
N\left(K, B_{2}^{n}\right) \leq \exp \left(A_{1} n\right) \text { and V.Rad. }(K) \geq a_{1}>0 .
$$

Then:

$$
N\left(K^{\circ}, B_{2}^{n}\right) \leq \exp \left(A_{2} n\right),
$$

where $A_{2} \leq A_{1}+\log \left(C / a_{1}\right)$, and $C>0$ is a universal constant.

Proof. Set $K_{s}=K \cap-K$. By the covering estimate of H. König and V. Milman [15], it follows that:

$$
N\left(K^{\circ}, B_{2}^{n}\right) \leq N\left(K_{s}^{\circ}, B_{2}^{n}\right) \leq C^{n} N\left(B_{2}^{n}, K_{s}\right) .
$$

Using standard volumetric covering estimates (e.g. [25, Chapter 7]), we deduce:

$$
N\left(K^{\circ}, B_{2}^{n}\right) \leq C^{n}\left(\frac{\operatorname{Vol}\left(B_{2}^{n}+K_{s} / 2\right)}{\operatorname{Vol}\left(K_{s} / 2\right)}\right) \leq C^{n} N\left(K_{s} / 2, B_{2}^{n}\right) \frac{\operatorname{Vol}\left(2 B_{2}^{n}\right)}{\operatorname{Vol}\left(K_{s} / 2\right)} .
$$

By a result of V. Milman and A. Pajor [20], it is known that $\operatorname{Vol}\left(K_{s}\right) \geq 2^{-n} \operatorname{Vol}(K)$, and hence:

$$
N\left(K^{\circ}, B_{2}^{n}\right) \leq(8 C)^{n} N\left(K, B_{2}^{n}\right) \operatorname{V} \cdot \operatorname{Rad} .(K)^{-n} \leq\left(8 C / a_{1}\right)^{n} \exp \left(A_{1} n\right),
$$

as required. 
Lemma 2.5. Let $L$ denote any compact set in $\mathbb{R}^{n}(n \geq 2)$, so that $N\left(L, B_{2}^{n}\right) \leq \exp \left(A_{1} n\right)$. If $U$ is uniformly distributed on $O(n)$, then:

$$
P\left(L \cap U(L) \subset A_{3} B_{2}^{n}\right) \geq 1-\exp \left(-A_{2} n\right),
$$

where $A_{2}=A_{1}+(\log 2) / 2$ and $A_{3}=C^{\prime} \exp \left(6 A_{1}\right)$, for some universal constant $C^{\prime}>0$.

Proof Sketch. Assume that $L \subset \cup_{i=1}^{\exp \left(A_{1} n\right)}\left(x_{i}+B_{2}^{n}\right)$. Set $R=4 C \exp \left(6 A_{1}\right)$, for some large enough constant $C>0$, and without loss of generality, assume that among all translates $\left\{x_{i}\right\},\left\{x_{i}\right\}_{i=1}^{N}$ are precisely those points lying outside of $R B_{2}^{n}$. Observe that for each $i=1, \ldots, N$, the cone $\left\{t\left(x_{i}+B_{2}^{n}\right) ; t \geq 0\right\}$ carves a spherical cap of Euclidean radius at most $1 / R$ on $S^{n-1}$. By the invariance of the Haar measures on $S^{n-1}$ and $O(n)$ under the action of $O(n)$, it follows that for every $i, j \in\{1, \ldots, N\}$ :

$$
P\left(U\left(x_{i}+B_{2}^{n}\right) \cap\left(x_{j}+B_{2}^{n}\right) \neq \emptyset\right) \leq \mu_{S^{n-1}}\left(B_{2 / R}\right),
$$

where $B_{\varepsilon}$ denotes a spherical cap on $S^{n-1}$ of Euclidean radius $\varepsilon$, and recall $\mu_{S^{n-1}}$ denotes the normalized Haar measure on $S^{n-1}$. When $\varepsilon<1 /(2 C)$, it is easy to verify that:

$$
\mu_{S^{n-1}}\left(B_{\varepsilon}\right) \leq(C \varepsilon)^{n-1},
$$

and so it follows by the union-bound that:

$P\left(L \cap U(L) \subset(R+1) B_{2}^{n}\right) \geq P\left(\forall i, j \in\{1, \ldots, N\} \quad U\left(x_{i}+B_{2}^{n}\right) \cap\left(x_{j}+B_{2}^{n}\right)=\emptyset\right) \geq 1-N^{2}(2 C / R)^{n-1}$.

Since $N \leq \exp \left(2 A_{1}(n-1)\right)$, our choice of $R$ yields the desired assertion with $C^{\prime}=5 C$.

It is also useful to state:

Lemma 2.6. For any density $g$ on $\mathbb{R}^{n}$ and $p \geq 1$ :

$$
Z_{p}^{+}(g) \subset 2^{1 / p} Z_{p}(g) \subset Z_{p}^{+}(g)-Z_{p}^{+}(g) .
$$

Proof. The first inclusion is trivial. The second follows since $a^{1 / p}+b^{1 / p} \geq(a+b)^{1 / p}$ for $a, b \geq 0$, and hence for all $\theta \in S^{n-1}$ :

$$
h_{Z_{p}^{+}(g)-Z_{p}^{+}(g)}(\theta)=h_{Z_{p}^{+}(g)}(\theta)+h_{Z_{p}^{+}(g)}(-\theta) \geq 2^{1 / p} h_{Z_{p}(g)}(\theta) .
$$

The next two theorems play a crucial role in our argument. The first is due to Paouris [22], and the second to the authors [14]:

Theorem (Paouris). With the same assumptions as in Theorem 1.1:

$$
W\left(Z_{p}(X)\right) \leq C \sqrt{p} \quad \forall p \in\left[2, c n^{\alpha / 2}\right] .
$$


Theorem (Klartag-Milman). With the same assumptions as in Theorem 1.1:

$$
\operatorname{V.Rad} .\left(Z_{p}(X)\right) \geq c \sqrt{p} \quad \forall p \in\left[2, c n^{\alpha / 2}\right] .
$$

We are finally ready to provide a proof of Proposition 2.1

Proof of Proposition [2.1. Let $p \in\left[2, c n^{\alpha / 2}\right]$, where $c>0$ is some small enough constant so that (2.2) and (2.3) hold. We will ensure that $c \leq 1$, so there is nothing to prove if $n=1$. By (2.1), Sudakov's entropy estimate (e.g. [25]) and (2.2), we have:

$N\left(Z_{p}^{+}(X) / \sqrt{p}, B_{2}^{n}\right) \leq N\left(2^{1 / p} Z_{p}(X) / \sqrt{p}, B_{2}^{n}\right) \leq \exp \left(\tilde{C} n W\left(2^{1 / p} Z_{p}(X) / \sqrt{p}\right)^{2}\right) \leq \exp (C n)$.

Note that by (2.1) and the Rogers-Shephard inequality [26], we have:

$$
2^{n / p} \operatorname{Vol}\left(Z_{p}(X)\right) \leq \operatorname{Vol}\left(Z_{p}^{+}(X)-Z_{p}^{+}(X)\right) \leq 4^{n} \operatorname{Vol}\left(Z_{p}^{+}(X)\right) .
$$

Consequently, the volume bound in (2.3) also applies to $Z_{p}^{+}(X)$ :

$$
\operatorname{V.Rad} .\left(Z_{p}^{+}(X)\right) \geq c_{1} \sqrt{p} .
$$

By Lemma 2.4, (2.4) and (2.5) imply that:

$$
N\left(\sqrt{p}\left(Z_{p}^{+}(X)\right)^{\circ}, B_{2}^{n}\right) \leq \exp \left(C_{2} n\right) .
$$

Consequently, Lemma 2.5 implies that if $U$ is uniformly distributed on $O(n)$, then for any $C_{1} \geq C_{2}+(\log 2) / 2$, there exists a $C_{3}>0$, so that:

$$
\mathbb{P}\left(Z_{p}^{+}(X)^{\circ} \cap U\left(Z_{p}^{+}(X)^{\circ}\right) \subset \frac{C_{3}}{\sqrt{p}} B_{2}^{n}\right) \geq 1-\exp \left(-C_{1} n\right),
$$

or by duality (since $T(K)^{\circ}=\left(T^{-1}\right)^{*}\left(K^{\circ}\right)$ for any linear map $T$ of full rank), that:

$$
\begin{aligned}
& \mathbb{P}\left(Z_{p}^{+}(X)+U\left(Z_{p}^{+}(X)\right) \supset C_{3}^{-1} \sqrt{p} B_{2}^{n}\right) \\
\geq & \mathbb{P}\left(\operatorname{conv}\left(Z_{p}^{+}(X) \cup U\left(Z_{p}^{+}(X)\right)\right) \supset C_{3}^{-1} \sqrt{p} B_{2}^{n}\right) \geq 1-\exp \left(-C_{1} n\right) .
\end{aligned}
$$

Lemma 2.3 now concludes the proof.

\section{$3 \quad$ Remaining Details}

We now complete the remaining (standard) details in the proof of Theorem 1.1. Proof of Theorem 1.1. 
1. For any $U \in O(n)$ and $t \geq 0$, observe that:

$$
\begin{aligned}
& 2 \max \left(\mathbb{P}\left(\left|\frac{X+U\left(X^{\prime}\right)}{\sqrt{2}}\right| \leq t\right), \mathbb{P}\left(\left|\frac{X-U\left(X^{\prime}\right)}{\sqrt{2}}\right| \leq t\right)\right) \\
\geq & \mathbb{P}\left(\left|\frac{X+U\left(X^{\prime}\right)}{\sqrt{2}}\right| \leq t\right)+\mathbb{P}\left(\left|\frac{X-U\left(X^{\prime}\right)}{\sqrt{2}}\right| \leq t\right) \\
= & \mathbb{P}\left(\frac{|X|^{2}+\left|X^{\prime}\right|^{2}}{2}+\left\langle X, U\left(X^{\prime}\right)\right\rangle \leq t^{2}\right)+\mathbb{P}\left(\frac{|X|^{2}+\left|X^{\prime}\right|^{2}}{2}-\left\langle X, U\left(X^{\prime}\right)\right\rangle \leq t^{2}\right) \\
\geq & \mathbb{P}\left(|X| \leq t \text { and }\left|X^{\prime}\right| \leq t \text { and }\left\langle X, U\left(X^{\prime}\right)\right\rangle \leq 0\right) \\
& +\mathbb{P}\left(|X| \leq t \text { and }\left|X^{\prime}\right| \leq t \text { and }\left\langle X, U\left(X^{\prime}\right)\right\rangle>0\right) \\
= & \mathbb{P}\left(|X| \leq t \text { and }\left|X^{\prime}\right| \leq t\right)=\mathbb{P}(|X| \leq t)^{2} .
\end{aligned}
$$

Similarly:

$$
2 \max \left(\mathbb{P}\left(\left|\frac{X+U\left(X^{\prime}\right)}{\sqrt{2}}\right| \geq t\right), \mathbb{P}\left(\left|\frac{X-U\left(X^{\prime}\right)}{\sqrt{2}}\right| \geq t\right)\right) \geq \mathbb{P}(|X| \geq t)^{2} .
$$

This is precisely the content of the first assertion of Theorem 1.1 .

2. Given $\theta \in S^{n-1}$, denote $Y_{1}=P_{\theta} Y_{+}^{U}, X_{1}=P_{\theta} X$ and $X_{2}=P_{\theta} U\left(X^{\prime}\right)$, where $P_{\theta}$ denotes orthogonal projection onto the one-dimensional subspace spanned by $\theta$. We have:

$$
\begin{gathered}
h_{Z_{p}\left(Y_{+}^{U}\right)}(\theta)=\left(\mathbb{E}\left|Y_{1}\right|^{p}\right)^{\frac{1}{p}}=\left(\mathbb{E}\left|\frac{X_{1}+X_{2}}{\sqrt{2}}\right|^{p}\right)^{\frac{1}{p}} \\
\leq \frac{1}{\sqrt{2}}\left(\left(\mathbb{E}\left|X_{1}\right|^{p}\right)^{\frac{1}{p}}+\left(\mathbb{E}\left|X_{2}\right|^{p}\right)^{\frac{1}{p}}\right)=\frac{1}{\sqrt{2}}\left(h_{Z_{p}(X)}(\theta)+h_{\left.Z_{p}(U(X))\right)}(\theta)\right) .
\end{gathered}
$$

Employing in addition (2.1), it follows that:

$$
Z_{p}^{+}\left(Y_{+}^{U}\right) \subset 2^{1 / p} Z_{p}\left(Y_{+}^{U}\right) \subset \frac{2^{1 / p}}{\sqrt{2}}\left(Z_{p}(X)+U\left(Z_{p}(X)\right)\right)
$$

and the second assertion for $Y_{+}^{U}$ follows since $Z_{p}(X) \subset C p^{\frac{1}{\alpha}} B_{2}^{n}$ by assumption. Similarly for $Y_{-}^{U}$.

3. Given a natural number $i$, set $p_{i}=2^{i}$. Proposition 2.1 ensures the existence of a constant $c>0$, so that for any $C_{1}>0$, there exists a constant $c_{1}>0$, so that for any $p_{i} \in\left[2, c n^{\frac{\alpha}{2}}\right]$, there exists a subset $A_{i} \subset O(n)$ with:

$$
\mu_{O(n)}\left(A_{i}\right) \geq 1-\exp \left(-C_{1} n\right)
$$

so that:

$$
\forall U \in A_{i} \quad Z_{p_{i}}\left(Y_{+}^{U}\right) \supset c_{1} \sqrt{p_{i}} B_{2}^{n}
$$


Denoting $A_{0}:=\cap\left\{A_{i} ; p_{i} \in\left[2, c n^{\frac{\alpha}{2}}\right]\right\}$, and setting $A=A_{0} \cap-A_{0}$, where $-A_{0}:=$ $\left\{-U \in O(n) ; U \in A_{0}\right\}$, it follow by the union-bound that:

$$
\mu_{O(n)}(A) \geq 1-2 \log \left(C_{2}+n\right) \exp \left(-C_{1} n\right) .
$$

By choosing the constant $C_{1}>0$ large enough, we conclude that:

$$
\mu_{O(n)}(A) \geq 1-\exp \left(-C_{3} n\right) .
$$

By construction, the set $A$ has the property that:

$$
\forall U \in A \quad \forall p_{i} \in\left[2, c n^{\frac{\alpha}{2}}\right] \quad Z_{p_{i}}\left(Y_{ \pm}^{U}\right) \supset c_{1} \sqrt{p_{i}} B_{2}^{n} .
$$

Using (1.6), it follows that:

$$
\forall U \in A \quad \forall p \in\left[2, c n^{\frac{\alpha}{2}}\right] \quad Z_{p}\left(Y_{ \pm}^{U}\right) \supset \frac{c_{1}}{\sqrt{2}} \sqrt{p} B_{2}^{n},
$$

thereby concluding the proof of the third assertion.

\section{References}

[1] L. Berwald. Verallgemeinerung eines Mittelwertsatzes von J. Favard für positive konkave Funktionen. Acta Math., 79:17-37, 1947.

[2] S. G. Bobkov. On concentration of distributions of random weighted sums. Ann. Probab., 31(1):195-215, 2003.

[3] Ch. Borell. Convex measures on locally convex spaces. Ark. Mat., 12:239-252, 1974.

[4] J. Bourgain and V. D. Milman. New volume ratio properties for convex symmetric bodies in $\mathbb{R}^{n}$. Invent. Math., 88:319-340, 1987.

[5] B. Fleury. Concentration in a thin euclidean shell for log-concave measures. $J$. Func. Anal., 259:832-841, 2010.

[6] M. Fradelizi. Contributions à la géométrie des convexes. Méthodes fonctionnelles et probabilistes. Habilitation à Diriger des Recherches de l'Université Paris-Est Marne La Vallée, 2008. http://perso-math.univ-mlv.fr/users/fradelizi.matthieu/pdf/HDR.pdf.

[7] R. J. Gardner. The Brunn-Minkowski inequality. Bull. Amer. Math. Soc. (N.S.), 39(3):355-405, 2002.

[8] B. Grünbaum. Partitions of mass-distributions and of convex bodies by hyperplanes. Pacific J. Math., 10:1257-1261, 1960. 
[9] O. Guédon and E. Milman. Interpolating thin-shell and sharp large-deviation estimates for isotropic log-concave measures. to appear in Geom. Func. Anal., arxiv.org/abs/1011.0943, 2010.

[10] C. Haberl. $L_{p}$ intersection bodies. Adv. Math., 217(6):2599-2624, 2008.

[11] B. Klartag. A central limit theorem for convex sets. Invent. Math., 168:91-131, 2007.

[12] B. Klartag. Power-law estimates for the central limit theorem for convex sets. $J$. Funct. Anal., 245:284-310, 2007.

[13] B. Klartag. On nearly radial marginals of high-dimensional probability measures. J. Eur. Math. Soc, 12:723-754, 2010.

[14] B. Klartag and E. Milman. Centroid bodies and the logarithmic Laplace Transform - a unified approach. submitted, arxiv.org/abs/1103.2985, 2011.

[15] H. König and V. D. Milman. On the covering numbers of convex bodies. In Geometrical aspects of functional analysis (1985/86), volume 1267 of Lecture Notes in Math., pages 82-95. Springer, Berlin, 1987.

[16] E. Lutwak and G. Zhang. Blaschke-Santaló inequalities. J. Differential Geom., 47(1):1-16, 1997.

[17] V. D. Milman. Inégalité de Brunn-Minkowski inverse et applications à la théorie locale des espaces normés. C. R. Acad. Sci. Paris Sér. I Math., 302(1):25-28, 1986.

[18] V. D. Milman. Entropy point of view on some geometric inequalities. C. R. Acad. Sci. Paris Sér. I Math., 306(14):611-615, 1988.

[19] V. D. Milman. Isomorphic symmetrizations and geometric inequalities. In Geometric aspects of functional analysis (1986/87), volume 1317 of Lecture Notes in Math., pages 107-131. Springer, Berlin, 1988.

[20] V. D. Milman and A. Pajor. Entropy and asymptotic geometry of non-symmetric convex bodies. Adv. Math., 152(2):314-335, 2000.

[21] V. D. Milman and G. Schechtman. Asymptotic theory of finite-dimensional normed spaces, volume 1200 of Lecture Notes in Mathematics. Springer-Verlag, Berlin, 1986. With an appendix by M. Gromov.

[22] G. Paouris. Concentration of mass on convex bodies. Geom. Funct. Anal., 16(5):1021-1049, 2006.

[23] G. Paouris. Small ball probability estimates for log-concave measures. To appear in Trans. Amer. Math. Soc., 2010. 
[24] G. Paouris. On the existence of supergaussian directions on convex bodies. preprint, 2011.

[25] G. Pisier. The volume of convex bodies and Banach space geometry, volume 94 of Cambridge Tracts in Mathematics. Cambridge University Press, Cambridge, 1989.

[26] C. A. Rogers and G. C. Shephard. The difference body of a convex body. Arch. Math., 8:220-233, 1957. 